\title{
Morphologic spectrum of gastrointestinal polyps and polypoid lesions - A five year study
}

\author{
Ramya Chitturi' ${ }^{1}$ I.V. Renuka ${ }^{2, *}$, P. Prema Latha ${ }^{3}$, R. Vaishnavi ${ }^{4}$, B. Manasa ${ }^{5}$ \\ ${ }^{1}$ Assistant Professor, ${ }^{2}$ Professor, ${ }^{3}$ Professor and HOD, ${ }^{4,5}$ Post Graduate, Dept. of Pathology, NRI Medical College, Andhra \\ Pradesh, India
}

*Corresponding Author: I.V. Renuka

Email: repriya56@gmail.com

\begin{abstract}
Introduction: Polyps begin as small elevations of the mucosa and polyps are most common in the colorectal region. The neoplastic polyps are important because they have a risk of transformation into malignancy. The role of the Pathologist is to identify any dysplastic changes and inform the clinician regarding the risk of development of carcinoma.

Materials and Methods: This is a retrospective analysis of polyps and polypoid lesions of 5 year duration from January 2013 to December 2017. The biopsies were stained with Haematoxylin \& Eosin and studied under light microscope.

Results: 107 cases of gastrointestinal polyps and polypoid lesions were included in our study. Hyperplastic polyps were the most common polyps found in our study. The most common site was rectum and stomach. Variety of lesions included in our study are multiple Peutz Jeghers (PJ) polyps, adenocarcinoma arising in PJ polyp, neuroendocrine tumours, and an angiomatous polyp.

Conclusion: Gastrointestinal polyps are divergent lesions. Differentiation of neoplastic polyps from non-neoplastic polyps is important in view of adenoma- carcinoma sequence developing in neoplastic polyps. Identification of hamartomatous polyps is also crucial since they are associated with syndromes and may unusually transform in to cancers. Pathologists play a key role in diagnosis of neoplastic polyps and polyps associated with syndromes. In our study hyperplastic polyps were most common with a variety of other lesions.
\end{abstract}

Keywords: Gastrointestinal polyps, Peutz Jeghers polyp, Peutz jeghers syndrome, Polypoid lesions.

\section{Introduction}

A gastrointestinal (GI) polyp is a discrete softtissue mass protruding into the lumen. ${ }^{1}$ Polyps may develop as a result of epithelial or stromal cell hyperplasia, inflammation, ectopia or neoplasm. ${ }^{2}$ Although endoscopic appearances of some polyps may be diagnostic, the term "polyp" should not be used for every discrete protrusion identified at endoscopy unless histopathologically confirmed. ${ }^{3}$ The presence of a systemic process that promotes the development of multiple gastro-intestinal polyps is termed 'polyposis. ${ }^{4}$

The polyp size and histological type are two important factors for development of cancer in a polyp. $^{5,6}$ Polyps with malignant potential are categorized as neoplastic polyps whereas polyps that are considered to have no or lesser cancer risk are categorized as non-neoplastic. The neoplastic polyps are important because they have malignant potential that represents a stage in the development of colorectal cancer.

Microscopic evaluation of polyps is very important especially to rule out foci of malignancy. ${ }^{6}$ It is essential to identify neoplastic polyps at a sufficiently early stage, where a simple endoscopic procedure to remove them can stop the development of colorectal cancer and prevent disease and death. ${ }^{7}$

The prevalence of colorectal adenomatous polyps varies widely from country to country. ${ }^{8}$ The prevalence of intestinal adenomas varies in different parts of the world and is common in westernized countries. It is very important to know the distribution of colorectal polyps in other countries, because it may affect the efficacy of screening modalities and also the prevalence of adenomas that are roughly equivalent to the risk of colorectal malignancies. ${ }^{9}$ The prevention of colorectal cancer can largely be attained by effective screening for adenomatous polyps before they develop the ability to invade. $^{10}$

\section{Material and Methods}

A retrospective observational study was done in department of Pathology. Data was retrieved from system for a period of 5 year duration from January 2013 to December 2017. All the polyps and polypoid lesions in gastrointestinal tract from oesophagus to anal canal were included. Haemorrhoids and fibroepithelial polyps were excluded from our study. Specimens include polypectomies and resection specimens that were received in $10 \%$ formalin. $3-4 \mu$ sections were cut and stained with H\&E. The lesions were categorised according to the site, nature and age. Immunohistochemistry was done wherever necessary. Data was analysed using Microsoft Excel sheets and presented as frequencies and percentages using tables.

\section{Results}

A total of 107 cases were included in the study. The commonly encountered polyps in our study were hyperplastic polyps and polypoid adenocarcinomas. The incidence of polyps was higher in males 72 $(67.2 \%)$ compared to females $35(32.7 \%)$. The age of the patients ranged from 4 yrs to $88 y$ rs. The mean age of patients in our study is $46.59 \mathrm{yr}$. 
We divided the patients into 9 age groups. Polyps were most commonly seen in the age group 61-70yrs and least commonly seen in 81-90yr age group (Table 1).

Table 1: Incidence of Nonneoplastic and Neoplastic polyps in relation to age

\begin{tabular}{|c|c|c|c|c|c|}
\hline S. No & Age group & Non neoplastic & Neoplastic & Total Number & Percentage \\
\hline 1 & $1-10 \mathrm{yrs}$ & 5 & 0 & 5 & $4.7 \%$ \\
\hline 2 & $11-20 \mathrm{yrs}$ & 3 & 2 & 5 & $4.7 \%$ \\
\hline 3 & $21-30 \mathrm{yrs}$ & 3 & 3 & 6 & $5.6 \%$ \\
\hline 4 & $31-40 \mathrm{yrs}$ & 5 & 5 & 10 & $9.3 \%$ \\
\hline 5 & $41-50 \mathrm{yrs}$ & 6 & 19 & 25 & $23.4 \%$ \\
\hline 6 & $51-60 \mathrm{yrs}$ & 8 & 13 & 21 & $19.6 \%$ \\
\hline 7 & $61-70 \mathrm{yrs}$ & 12 & 16 & 28 & $26.2 \%$ \\
\hline 8 & $71-80 \mathrm{yrs}$ & 2 & 3 & 5 & $4.7 \%$ \\
\hline 9 & $81-90 \mathrm{yrs}$ & 0 & 2 & 2 & $1.9 \%$ \\
\hline & Total & 44 & 63 & 107 & $100 \%$ \\
\hline
\end{tabular}

Among the $1-10 y r s$ age group, all the polyps were histologically non neoplastic polyps. Up to $40 \mathrm{yrs}$, nonneoplastic polyps were more common or equal to

Table 2: Distribution of polyps according to site

\begin{tabular}{|l|c|c|c|}
\hline S. No & Site & Number $(\mathbf{n}=\mathbf{1 0 7})$ & Percentage \\
\hline 1 & Stomach & 34 & $31.8 \%$ \\
\hline 2 & Rectum & 28 & $26.2 \%$ \\
\hline 3 & Colon & 20 & $18.7 \%$ \\
\hline 4 & Esophagus & 5 & $4.7 \%$ \\
\hline 5 & Ileum & 4 & $3.7 \%$ \\
\hline 6 & Recto sigmoid & 3 & $2.8 \%$ \\
\hline 7 & Anal canal & 3 & $2.8 \%$ \\
\hline 8 & Duodenum & 3 & $2.8 \%$ \\
\hline 9 & Caecum & 2 & $1.9 \%$ \\
\hline 10 & Gastroesophageal junction & 2 & $1.9 \%$ \\
\hline 11 & Jejenum & 1 & $0.9 \%$ \\
\hline 12 & Anorectum & 1 & $0.9 \%$ \\
\hline 13 & Periampullary region & 1 & $0.9 \%$ \\
\hline
\end{tabular}

The most common site for polyps was stomach (31.8\%) followed by rectum $(26.2 \%)$ and colon $(18.7 \%)$ (Table 2). Neuroendocrine tumours were most commonly found in rectum and stomach.

On histology, most common polyps were hyperplastic polyps, followed by adenocarcinomas and adenomatous polyps (Table 3 ). The size of most of the polyps was $<1 \mathrm{~cm}$. Mild to moderate dysplasia was identified in most of the adenomatous polyps.7 cases of adenocarcinomas were associated with polyps of which, 6 cases of adenocarcinomas were associated with adenomatous polyps and 1 was found to be arising from PJ polyp. The other polypoid lesions encountered in our study were lipomatous polyp, Brunner's gland adenoma, inflammatory fibroid polyp, Angiomatous polyp, multiple PJ polyps, melanoma, and a sub mucosal GIST. the number of neoplastic polyps but after $40 \mathrm{yrs}$, the incidence of neoplastic polyps is greater than nonneoplastic polyps. After 80yrs, all the polyps in our study were neoplastic polyps.
Table 3: Histological types of polyps

\begin{tabular}{|l|c|c|}
\hline S. No & Histologic type & $\begin{array}{c}\text { Number } \\
(\mathbf{n = 1 0 7})\end{array}$ \\
\hline 1. & Hyperplastic polyps & 29 \\
\hline 2. & Adenocarcinoma & 25 \\
\hline 3. & Adenomatous polyps & 16 \\
\hline 4. & Juvenile polyps & 8 \\
\hline 5. & Lipomatous polyps & 5 \\
\hline 6 & Neuroendocrine tumour & 4 \\
\hline 7. & Inflammatory polyps & 4 \\
\hline 8. & Fundic gland polyps & 3 \\
\hline 9. & Squamous cell carcinoma & 3 \\
\hline 10. & Hamartomatous polyps & 2 \\
\hline 11. & Brunner gland adenoma & 1 \\
\hline 12. & Inflammatory fibroid polyp & 1 \\
\hline 13. & Angiomatous polyp & 1 \\
\hline 14. & Multiple PJ polyps & 1 \\
\hline 15. & Adenocarcinoma arising in PJ & 1 \\
\hline & polyp & 1 \\
\hline 16. & Melanoma & 1 \\
\hline 17. & Poorly Differentiated Carcinoma & 1 \\
\hline 18. & Submucosal GIST & 1 \\
\hline
\end{tabular}


In a case of a rectal polypoid lesion, based on histology the differential diagnosis was poorly differentiated carcinoma and neuroendocrine tumour. Synaptophysin and pancytokeratin (panCK) were done. There was diffuse cytoplasmic positivity in the cells with Synaptophysin and dot like positivity with panCK. So it was diagnosed as neuroendocrine tumour. In an another case, 120 adenomatous polyps were identified in colon. It was diagnosed as Familial Adenomatous Polyposis (FAP) (Fig. 1).
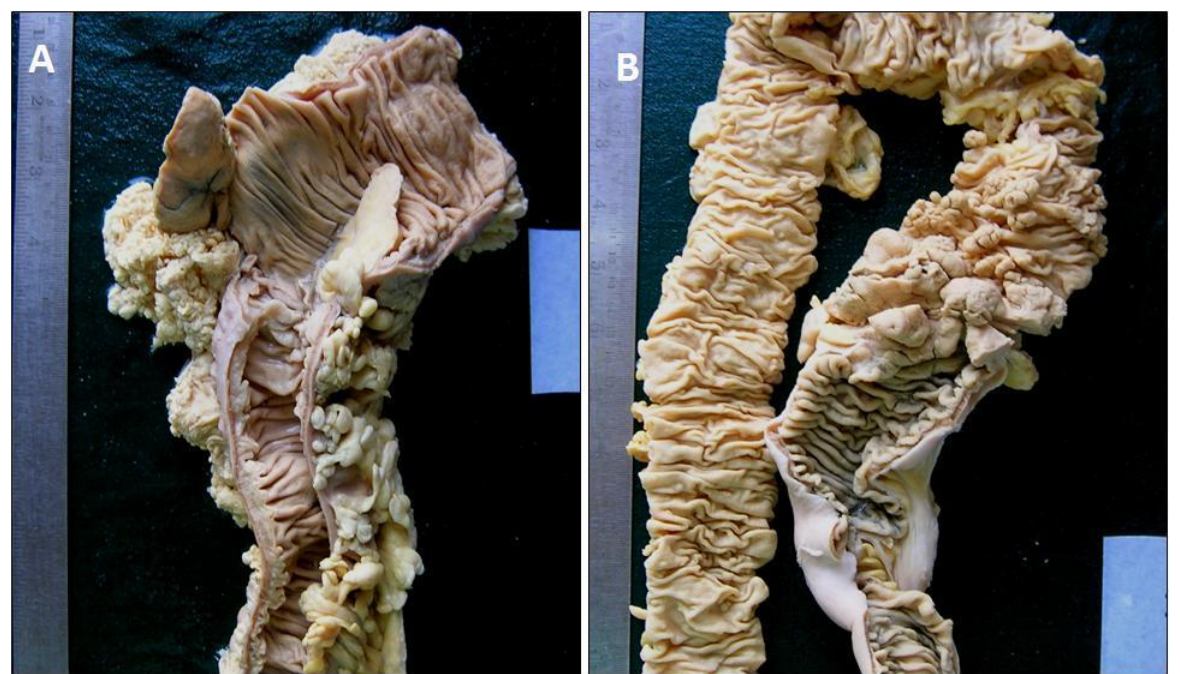

Fig. 1 A): Gross picture: lipomatous polyp of colon; B): Gross picture of Familial adenomatous polyposis with multiple variably sized polyps in colon

3 PJ polyps were diagnosed histologically in colon, so it was informed to clinician as a case of Peut Jeghers Syndrome (PJS). A case of rectal adenocarcinoma arising in a PJ polyp clinically revealed prominent mucocutaneous pigmentation of the

lips and oral cavity that were not identified by the surgeon initially (Fig. 2, 3). Hence it was diagnosed as PJS and the patient is on follow up. Hence we emphasize the need for complete clinical details of patients along with the specimens.
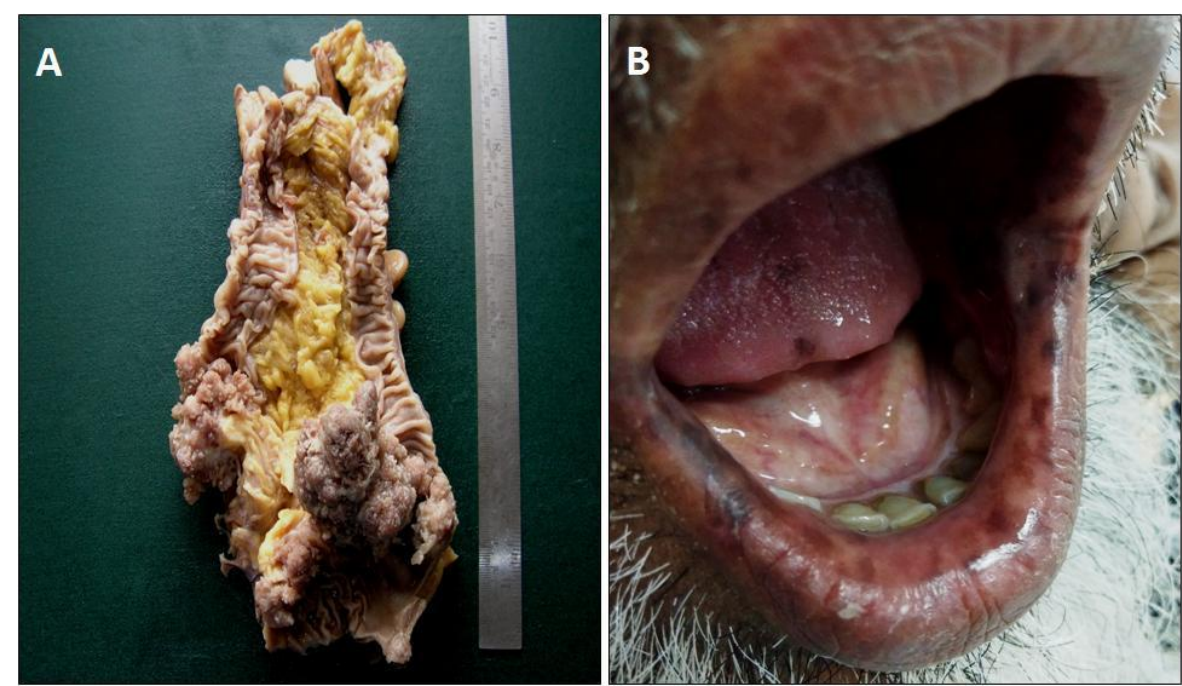

Fig. 2 A): Gross picture: Peutz Jeghers syndrome with multiple polyps in colon and with adenocarcinomatous transformation; B): Mucocutaneous pigmentation in PJ Syndrome of the same case 


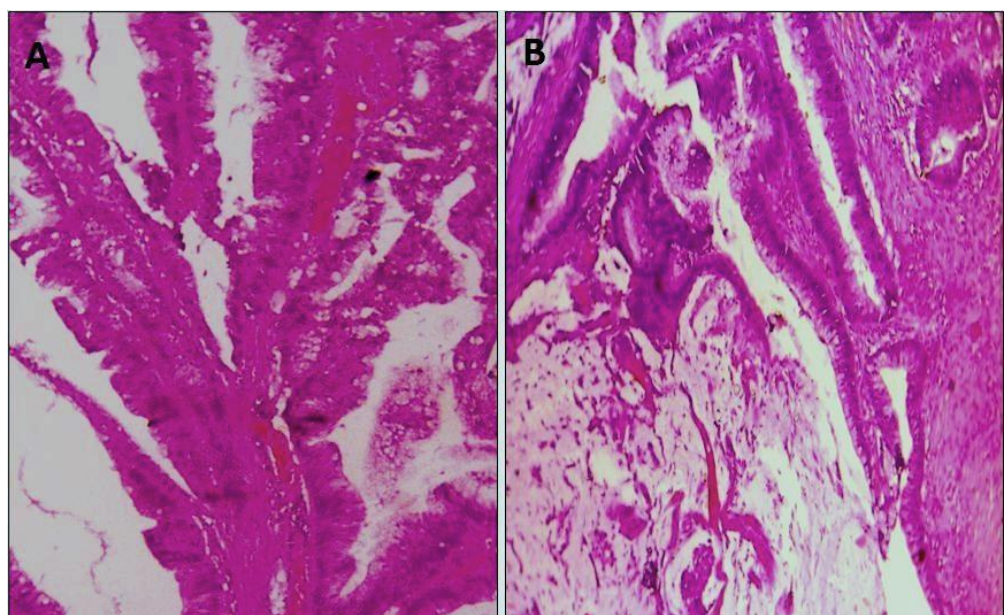

Fig. 3 A): PJ polyp with broad bands of muscularis mucosa (H\&E x100); B): PJ polyp with mucinous adenocarcinoma (H\&E x100)

\section{Discussion}

Gastrointestinal polyps can develop throughout the gastrointestinal tract, in esophagus, stomach, small intestine and colon. Most of the studies have reported higher incidence of polyps in colon.

Syndromic associations were seen in 3 cases in our study. They were 2 cases of PJS and 1 case of FAP. The PJ polyp constitutes a rare subtype with a characteristic histological appearance showing tree like branching of smooth muscle cells and lobular organization of colonic crypts. ${ }^{11}$ Glandular disorganization and epithelial misplacement simulating invasion should not be confused with malignancy. ${ }^{12}$ Peutz ${ }^{13}$ and Jeghers ${ }^{14}$ et al first described PJS. Identification of PJ polyps cannot be underestimated because the patient and their family are at an increased risk of developing carcinoma of the pancreas, cervix, breast, ovaries and testis. ${ }^{15}$

In our study, the incidence of gastrointestinal polyps was higher in males $72(67.2 \%)$ compared to females $35(32.7 \%)$. This is in correlation with other studies by Mirzaie et al ${ }^{6}$ Gentiana et $\mathrm{al}^{16}$ and Deepa Thomas et al. ${ }^{10}$

Polyps were most commonly seen in the age group 61-70yrs $(26.2 \%)$ in our study, whereas the polyps were more prevalent between $70-80$ year age group in a study by Mirzaie et al (25.2\%). ${ }^{6}$

Stomach $(31.8 \%)$ and rectum $(26.2 \%)$ were the most common sites for GI polyps in our study. Sharifi et al performed study on pediatric Iranian population, in which $75.8 \%$ of polyps were in colon and $24.1 \%$ were in stomach. ${ }^{17}$ Colon and sigmoid $(74.2 \%)$ were sites of most of GI polyps in study by Mirzaie et al, ${ }^{6}$ Recto sigmoid in a study by Gentiana et $\mathrm{al}^{16}$ and Rectum (39\%) in a study by Deepa Thomas et al. ${ }^{10}$ Gurung et al reported large intestine as the commonest site for the polyps $(83.6 \%){ }^{18}$

The size of most of the polyps in our study was less than $1 \mathrm{~cm}$. The incidence of invasive carcinoma in a polyp is classically considered to depend on its size and histological type. The respective risks of carcinoma are less than $5 \%$ in a tubular adenoma smaller than one $\mathrm{cm}$ and may reach $50 \%$ in a large villous adenoma which is more than $2 \mathrm{~cm}$.

Nonneoplastic polyps were most common in our study 44 cases $(41.1 \%)$. This is in contrast to other studies by Germizadeh et al, ${ }^{1}$ Mirzaie et $\mathrm{al}^{6}{ }^{6}$ and Gentiana et al. ${ }^{16}$

Geramizadeh et al reported adenomatous polyps in $603(60.9 \%)$ cases and were located in the recto sigmoid area. ${ }^{1}$ Mirzaie et al also reported adenomatous polyp as the most common histologic type. ${ }^{6}$ The most common types of polyps were adenomatous polyps 300 (72.99\%), followed by hyperplastic $69(16.78 \%)$ in study by Gentiana et al. ${ }^{16}$

Esophageal polyps are rare $^{19}$ and Mirzaie et al had only 1 case in 210 gastrointestinal polyps which was of hyperplastic type. ${ }^{6}$ We had 4 esophageal polypoid lesions in our study and most of them were malignancies.

Incidence of gastric polyps in our study was 34 (31.8\%) and most of them are hyperplastic polyps. The incidence of gastric polyps was $2 \%$ in 2630 patients who underwent upper GI endoscopic examination in a study by Rasim Gencosmanoglu et al. ${ }^{20}$ The most frequently encountered histopathologic subtype of gastric polypoid lesions in their study was hyperplastic polyp which was diagnosed in $46 \%$ of the lesions, followed by foveolar hyperplasia, fundic gland polyp.

The incidence of malignancy in polyps is $9 \%$ in study by Deepa Thomas et al. ${ }^{10}$ In our study incidence of malignancy in polyps was $6.5 \%$. The risk of development of carcinoma in FAP is $1-6 \%$ by age $21 \mathrm{yrs}$ and $>90 \%$ of untreated patients develop carcinoma by age 50years. In carriers with MLH1 and MSH2 mutations in lynch syndrome, the risk of development of cancer is $>80 \%$ during their life time. ${ }^{15}$ The risk of development of carcinoma in PJS is 3-6\%. 


\section{Conclusion}

In our study, age is one of the important risk factor as the incidence of adenomas and malignancy found to be higher after 4oyears. Size of the polyp, degree of dysplasia are important factors, which we have included in our study. Males have higher incidence of GI polyps than females. Hyperplastic polyps were most common in our study and stomach was the most common site. Hamartomatous polyps should be evaluated with complete clinical history as they may show syndromic association and may rarely turn malignant. Immunohistochemistry aided in the diagnosis of some polypoid lesions like neuroendocrine tumour. We have diagnosed PJS in one case and alerted the clinicians. Pathologists play a key role in diagnosis of dysplasia in the polyps and polyps associated with syndromes.

Ethical Clearance: has been taken from institutional review board.

\section{Funding: None}

\section{Competing Interests: None}

\section{References}

1. Geramizadeh B, Jahromi MK. Pathology of Colorectal Polyps: A Study from South of Iran. Ann Colorectal Res. 2013;1:59-61.

2. Kumar V, Abbas A K., Aster J C. Robbins and Cotran pathologic basis of disease. $9^{\text {th }}$ edition. Philadelphia: Elsevier/ Saunders; 2015 pg 769.

3. Oberhuber G, Stolte M. Gastric polyps: An update of their pathology and biological significance. Virchows Arch. 2000;437:581-0.

4. Noam Shussman and Steven D. Wexner. Colorectal polyps and polyposis syndromes. Gastroenterology Report 2, 2014;1-15.

5. Aldridge AJ, Simson JN. Histological assessment of colorectal adenomas by size. Are polyps less than $10 \mathrm{~mm}$ in size clinically important? Eur J Surg. 2001;167(10):777-81.

6. Mirzae AZ, Abolhasani M, Moghaddam RM, Kabivar M. The frequency of gastrointestinal polyps in Iranian population. Iranian Journal of Pathology. 2012;7:183-9.

7. Giacosa A, Frascio F, Munizzi F. Epidemiology of colorectal polyps. Tech Coloproctol 8 Suppl. 2:2008; s243-247.

8. Patel K, Hoffman NE. The anatomical distribution of colorectal Polyps at colonoscopy. J Clin Gastroenterol. 2001;33:222-5.

9. Kunjumon DT, Glaxon JA, Pushpalatha PK. A histopathological study of 23 cases of benign epithelial polyps of intestine. IJBR. 2014;5(2).

10. Tse JY, Wu S, Shinagare SA, Lauwers GY, Yilmaz O, Wu CL, Deshpande V. Peutz-Jeghers syndrome: a critical look at colonic Peutz-Jeghers polyps. Mod Pathol. 2013;26:1235-40.

11. John R Goldblum, Laura W.Lamps, Jesse K.McKenney, Jeffrey L. Myers. Rosai and Ackerman's Surgical Pathology $11^{\text {th }}$ edition. Philadelphia: Elsevier/ Saunders; 2018 pg673-4.

12. Peutz JLA. Very remarkable case of familial case of polyposis of mucous membrane of intestinal tract and nasopharynx accompanied by peculiar pigmentations of skin and mucous membrane. Ned Maandschr Geneeskd. 1921;10:134-46.

13. Jeghers H, Mckusick VA, Katz KH. Generalized intestinal polyposis and melanin spots of the oral mucosa, lips and digits; a syndrome of diagnostic significance. New Engl J Med. 1949;241:1031-6.

14. Bosman FT, Carneriro F, Hruban RH, Theise N D.WHO classification of tumours of the digestive system. 4th edition. IARC: Lyon, France; 2010 pg 169.

15. Gentiana C, Altin C, Arben B, Mehdi A. Histopathological Characteristics of Colon Polyps - A Population-based Study in Tirana, Albania. J Gastrointest Dig Syst. 2015;5:271.

16. Sharifi D, Akglagh M. Review of 145 patients with gastrointestinal polyps of Ghaem Medical Center, Mashhad. Med J Mashad University Medl Sci. 2000;69(43):25-32.

17. Gurung P, Hirachand S, Pradhanang S and Lama S. A histopathological study of Gastrointestinal polyps in Tertiary care hospital, Nepal. Journal of institute of medicine. 2014,36(1):64-68.

18. Abraham SC, Singh VK, Yardley JH, Wu TT. Hyperplastic polyps of the esophagus and esophagogastric junction: histologic and clinicopathologic findings. Am J Surg Pathol. 2001;25(9):1180-7.

19. Gencosmanoglu R, Sen-Oran E, Kurtkaya-Yapicier O, Avsar E, Sav A, Tozun N. Gastric polypoid lesions: analysis of 150 endoscopic polypectomy specimens from 91 patients. World J Gastroenterol. 2003;9(10):2236-9. 